\title{
¿FEMINISMO DE LA IGUALDAD Y FEMINISMO DE LA DIFERENCIA?
}

\author{
ELENA NÁJERA \\ Universidad de Alicante
}

\section{Introducción}

Solidaria con la trayectoria del mundo contemporáneo, la teoría feminista ha vivido en las últimas décadas una agitada época ocupada en la revisión de su propia tradición y dedicada así mismo a la ampliación de intereses y multiplicación de enfoques. Una de las coordenadas que ha permitido ordenar conceptual, y también políticamente, este rico y sin duda complejo panorama es la oposición establecida entre el feminismo de la igualdad y el feminismo de la diferencia. Se trata de una tipología clásica, y especialmente cultivada en el contexto académico español, que se hace cargo del intenso debate mantenido entre quienes sitúan la reflexión de género en la estela del proyecto ilustrado, comprometiéndola con los valores igualitaristas, universalistas y humanistas que lo vertebran, y quienes pretenden, por el contrario, desvincularla críticamente de este discurso acusándolo de androcentrismo. Este segundo frente tiene una significativa afinidad con el pensamiento postmoderno y, de idéntica manera que éste no se deja reducir a una única fórmula, el rótulo feminismo de la diferencia se ha visto obligado a acoger movimientos muy diversos, y a veces un tanto dispares, que guardan a su vez relaciones varias con los planteamientos básicos del feminismo de la igualdad. Por su parte, esta otra denominación está lejos de ser unívoca, exigiendo así mismo precisiones y subdivisiones capaces de dar cuenta de la totalidad de sus alcances y desarrollos.

En cualquier caso, la confrontación de ambos tipos de feminismos no puede ni debe tomarse a estas alturas como una disyuntiva definitoria, algo que la descubriría inmediatamente como un falso dilema. En la misma medida que muchas dicotomías polémicas, ésta ha tenido un fecundo recorrido 
que ha servido para orientar y agitar dialécticamente las corrientes feministas, pero, como todas las clasificaciones, ésta acusa también sus límites. Unos límites que quizás estén ya sobrepasados por el curso de los actuales estudios de género y hayan de dejar paso -hayan, de hecho, dejado paso ya- a otras etiquetas o estilos de etiquetar más útiles. De ahí los signos de interrogación que acompañan el título de este número monográfico y que pretenden invitar a una reflexión sobre la propia identidad de la teoría feminista y su preocupación por concebirse a sí misma a la altura de las exigencias intelectuales y políticas del presente.

De acuerdo con lo dicho, las autoras que afrontan en estas páginas la cuestión han coincidido mayormente al señalar que el recorrido de la contraposición entre esas dos modalidades tradicionales del feminismo está agotado y que se hace necesario explorar un camino alternativo que se aleje de esencialismos y que ha de desbordar forzosamente categorizaciones poco flexibles. Por ello, las perspectivas que recogen los artículos aquí reunidos permiten no sólo reconstruir la historia de esa confrontación y de sus circunstancias culturales, éticas y políticas, sino también bosquejar los nuevos esquemas conceptuales por los que el pensamiento feminista quiere discurrir. Y éste parece dispuesto a asumir a modo de nueva seña un prefijo tan cargado de expectativas como de imprecisiones. Parece que nos encontramos, en efecto, en un tiempo de Postfeminismo, un tiempo en el que los estudios sobre la mujer quizás deban confiar en otros criterios más allá de las viejas apelaciones a la igualdad y la diferencia, un tiempo con voluntad de futuro que, sin duda, también ha de merecer una cuidadosa consideración.

Los tres primeros textos que abren el índice abordan la problemática que nos ocupa con visión crítica y de conjunto, en un intento de trazar el mapa del pensamiento feminista contemporáneo. Sonia Reverter presenta el tema en «El feminismo: más allá de un dilema ajeno», artículo en el que se reconocen y repasan las ganancias del debate mantenido entre el feminismo de la igualdad y de la diferencia en los años setenta y ochenta de cara a la reflexión de género. Ésta exigiría, sin embargo, avanzar hacia otros enfoques en los que la igualdad y la libertad no cuenten como un dilema irresoluble, sino como dos aspectos ineludibles que no pueden sacrificarse uno a otro. Se trata, por tanto, de ampliar y diversificar la agenda del feminismo, un feminismo que en su tercera ola ha de seguir aspirando a ser una praxis transformadora capaz de generar un mundo mejor.

De una manera rigurosa y exhaustiva, Elvira Burgos proporciona la cartografía de los nuevos feminismos en «Cruzando líneas. Trazando conexiones». Como el mismo título del artículo se encarga de indicar, para esta autora el 
feminismo de la igualdad y el de la diferencia, lejos de señalar los únicos caminos del feminismo actual, se apoyan en una división que han transcendido y hecho inoperante otras posiciones de última generación. Éstas obtienen precisamente su fuerza en la articulación profunda y compleja de diferentes categorías y en la capacidad de entrecruzar los resultados de las distintas teorías feministas y de valorar su interdependencia. En este sentido, la autora presta especial atención a los planteamientos lesbiano, postestructuralista y queer en sus diversas versiones, con la conciencia de que el feminismo es, en cualquier caso, una teoría viva y en devenir mal avenida con tipologías rígidas.

En tercer lugar, en «Dos dogmas del feminismo» Carmen González desarrolla un estudio crítico sobre las expectativas que abre el giro postfeminista como modo de sortear la aporía a la que aboca el dilema del feminismo de la igualdad y de la diferencia. Para ello, parte del análisis de las dos creencias dogmáticas que, a su entender, lo sustentan: la afirmación de que la alteridad de la mujer con respecto al varón es responsable de su subordinación y la idea de que esta alteridad exige o bien superarla, luchando por la igualdad, o bien vindicarla como algo específico y constitutivo de lo femenino. Ambas estrategias han consentido el esencialismo y han caído en la ansiedad clasificatoria, algo que pretende corregir precisamente el postfeminismo. Como ejemplo, la autora examina la propuesta de Sylviane Agacinski apreciando sus posibilidades, pero advirtiendo así mismo de su peligroso desvío hacia lo privado que evita el compromiso público.

Una vez ganada perspectiva y dibujado el horizonte de la reflexión feminista contemporánea, los artículos que vienen a continuación se presentan como una ocasión para revisar los temas y problemas asociados a la distinción entre el feminismo de la igualdad y el feminismo de la diferencia y para repasar sus hitos históricos. En esta línea, las dos colaboraciones siguientes se centran en la segunda de estas corrientes considerándola tal y como ha sido encauzada e impulsada en círculos italianos. Aránzazu Hernández Piñero aborda en «Igualdad, diferencia: genealogías feministas» la génesis del pensamiento y la práctica de la diferencia sexual prestándole especial atención a Luce Irigaray y a la Librería de Mujeres de Milán. Su exposición pretende mostrar el sentido filosófico y político de la crítica al modelo igualitarista y analizar, como contrapartida, la propuesta política de articular mediaciones sexuadas como una forma de vinculación y elaboración femenina del mundo que ayuda a la expresión de la libertad, siendo ésta precisamente, a su entender, la cuestión decisiva. El texto está interesado en dar cuenta de la riqueza y diversidad del pensamiento de la diferencia sexual pero con la intención de 
advertir y reivindicar, en definitiva, la fecundidad que tienen los conflictos en el seno de la teoría feminista.

Por su parte, Annalisa Mirizio en «¿Adónde conduce la exaltación de lo femenino? Logros y límites políticos del pensamiento de la diferencia sexual italiano» asume la tarea de investigar en qué medida esta propuesta políticoteórica puede realmente configurarse como un discurso alternativo al feminismo de la igualdad capaz de abarcar y dar voz al sujeto mujer. Para ello, la autora estudia la formación de la llamada "política del deseo» a partir de la alianza conceptual entre el planteamiento de Luce Irigaray y el de Carla Lonzi, reconstruyendo así mismo el intenso debate que ha generado y que es el que, poniendo en evidencia precisamente sus límites, ha permitido recoger los mejores frutos del pensamiento de la diferencia sexual. Estos límites señalan la necesidad de que el feminismo, más allá de maneras dogmáticas y sin caer en la tentación narcisista de rivalizar con el discurso androcéntrico, siga siendo una interrogación crítico-reflexiva dispuesta a encajar sus propias contradicciones y fracasos.

La siguiente pareja de artículos permite recuperar dos momentos del diálogo mantenido en las últimas décadas entre el feminismo de la igualdad y el feminismo de la diferencia. En «¿En los umbrales del género? Beauvoir, Butler y el feminismo ilustrado» Leticia Sabsay examina ciertas derivas de una categoría medular para el proyecto feminista ilustrado, como es la de género, con el fin de señalar algunos de los actuales desafíos que se le plantean. Para ello, se centra en primer lugar en la lectura que Judith Butler hace de Simone de Beauvoir para, en un segundo momento, ocuparse de la crítica dirigida contra la perspectiva de la performatividad genérica a propósito de la cuestión de «cómo se deviene mujer». En este contexto, la autora se detiene en el debate en torno al constructivismo radical que parecería tener su origen en el postestructuralismo, aunque cabría revisar el carácter constructivista que podría reclamar así mismo la noción de género tal y como la tematiza Beauvoir.

La autora de El segundo sexo coprotagoniza también el siguiente artículo: «Variaciones feministas en torno a la inmanencia y la trascendencia. Simone de Beauvoir, Luce Irigaray y la «política de lo simbólico»». Aquí Mercedes López Jorge realiza de la mano de los conceptos «trascendencia» e «inmanencia» un recorrido por la historia del feminismo que distingue tres capítulos fundamentales. Tres capítulos que coincidirían en el reconocimiento del carácter constructivista del género, aunque discreparían a la hora de precisar cómo se ha de realizar esa construcción a través de la acción de las mujeres. El primero de ellos corresponde a Simone de Beauvoir y a su exaltación de la trascendencia frente a la condena de la inmanencia. En el segundo tiene 
cabida Luce Irigaray con su concepto de «trascendental sensible», que hace de la trascendencia algo absolutamente deudor de la inmanencia. Y el tercero cede la palabra el feminismo italiano de la diferencia sexual y su «política de lo simbólico», para la cual la trascendencia real, la que puede hacer política, late y se dirime en lo inmanente.

Ganando en detalle, el índice ofrece a continuación dos aproximaciones particulares, desde las circunstancias españolas y de América del Sur respectivamente, al debate entablado entre el feminismo de la igualdad y de la diferencia. María Isabel Corbí Sáez ilustra la cuestión reflexionando sobre la suerte de la obra de Beauvoir en nuestro país. En «Simone de Beauvoir en España: sus obras traducidas y su recepción en la prensa» recuerda cómo la presencia de la pensadora francesa comenzó a ser completa y definitiva en el mundo editorial y periodístico español a partir de la Transición. Sin embargo, el artículo puntualiza cómo en el ámbito de los Estudios Literarios ha pesado una cierta desconsideración motivada por las acusaciones de misoginia y de ensalzamiento de los valores masculinos lanzadas fundamentalmente por el potente y celebrado feminismo postmoderno francés. Esta situación, a entender de la autora, parece, no obstante, haber dado de la mano de Julia Kristeva y su «(Re)descubrimiento» de Simone de Beauvoir un giro capaz de volver a poner este nombre al servicio de la causa de la mujer.

Por su parte, M. Luisa Femenías en «Notas acerca de un debate en América del Sur sobre la dicotomía «Feminismo: ¿igualdad o diferencia?» sitúa los antecedentes teóricos de la problemática que trata en Uruguay y en Argentina a principios del siglo XX. A partir de aquí, analiza la expansión en América Latina en los años noventa de un pensamiento reivindicativo de las diferencias de las mujeres que rechaza el modelo igualitarista ilustrado por ser partícipe de la ideología de las «feministas blancas» y/o del «Primer mundo» para adoptar, adaptándolas, las teorías francesas del discurso así como una pluralidad de análisis provenientes del pensamiento postcolonial, el multicultural y de la subalternidad. El texto muestra cómo, asumiendo el problema de la «identidad» y de la mano de las políticas del reconocimiento, en la reflexión feminista de América del Sur se entrelazan el interés étnico-cultural y el de sexo-género.

El volumen se completa con dos contribuciones que insisten en las consecuencias prácticas -éticas y jurídicas- de la compleja y aporética polémica entre la igualdad y la diferencia que nos ocupa. Avanzando en esta dirección, Cristina Guirao Mirón en «Modernidad y postmodernidad en el feminismo contemporáneo» examina dos críticas fundamentales del pensamiento feminista a la modernidad: la separación de los espacios privado/público y el 
universalismo normativo. Para esta autora, la separación de espacios privado/ público tiene su origen en la modernidad, se consolida tras la Revolución Industrial y en nuestras actuales sociedades es la causa de la desigualdad estructural de los géneros. El universalismo normativo, de raíz deontológica, constituye, por su parte, la crítica más importante de la postmodernidad al proyecto ilustrado.

Como punto final, el trabajo de Malena Costa «El debate igualdad/diferencia en los feminismos jurídicos» facilita una aproximación a nuestro tema desde la óptica del derecho. En estas páginas, se repasan las principales perspectivas del feminismo jurídico, revisándose cómo se definen y problematizan en este contexto las perspectivas de género. Así, la autora establece una clasificación dialéctica entre feminismos de la igualdad -que englobarían el feminismo liberal clásico, el feminismo liberal social y el feminismo socialista-, feminismos de la diferencia interesados en la crítica a la neutralidad del derecho -que acogerían posturas como las del feminismo radical y el feminismo cultural-y los feminismos deconstructivistas o postfeminismos que, haciéndose eco de puntos de vista como los que representan los lesbofeminismos, las feministas negras, los queer studies o los relatos de la otredad o subalternidad, someten a discusión la pertinencia de la división igualdad/diferencia y ponen en cuestión la utilidad de la propia pervivencia del derecho.

Llegando ya al final de esta presentación, puede afirmarse que la nómina de pensadoras y pensadores que cubren los artículos que componen el presente número de Feminismo/s es amplia. De Simone de Beauvoir a Judith Butler, pasando por Luce Irigaray, Alessandra Bocchetti, Lia Cigarini, Teresa de Lauretis, Carla Lonzi, Monique Wittig, Rosi Braidotti, Gayle Rubin, Carole Pateman, Nancy Frazer, Celia Amorós, Luisa Muraro, Sylviane Agacinski, Gilles Deleuze, Jürgen Habermas... Estos y otros nombres han ayudado a nuestras autoras a dibujar un mosaico de perspectivas originales que valoran tanto las posibilidades como los límites de la discusión mantenida en las últimas décadas entre el feminismo de la igualdad y el feminismo de la diferencia. A modo de reflexión final, podría decirse que, en conjunto, los textos aquí convocados no dudan en señalar las dificultades y perplejidades así como las resbaladizas paradojas con las que no puede dejar de encontrarse la teoría feminista, una teoría que no sólo aspira a la coherencia conceptual sino, sobre todo, a la relevancia práxica.

A las autoras sólo queda agradecerles su amable colaboración y su honestidad intelectual. 